\title{
Análise virtual de usabilidade com idosos: estudo de distanciamento de botões de um controle remoto
}

\author{
Virtual usability analysis with elderly users: study of a remote control keys spacing \\ Mayara Ramos \\ Doutoranda em Engenharia de Produção pela Universidade Federal de Santa Catarina (UFSC) - \\ mayara.ramos@gmail.com \\ Aníbal Alexandre Campos
}

Doutorado em Engenharia Mecânica pela Universidade Federal de Santa Catarina (UFSC). Professora da Universidade do Estado de Santa Catarina (UDESC) - alexandre.campos@udesc.br

Alexandre Amorim dos Reis

Doutorado em Engenharia de Produção pela Universidade Federal de Santa Catarina (UFSC). Professor da Universidade do Estado de Santa Catarina (UDESC) - alexandre.reis@pq.cnpq.br

\section{Resumo}

O envelhecimento populacional trás consigo a necessidade de atender as limitações das habilidades dessa população. Para isso é necessário ter projetos de artefatos e serviços que foquem nesses usuários e as suas condições físicas e cognitivas. Neste trabalho é apresentado uma proposta para a inclusão destes usuários por meio de um teste virtual de usabilidade na plataforma do software SolidWorks ${ }^{\circledR}$. Esse teste buscou avaliar se o distanciamento dos botões de um modelo de controle remoto está adequado para os usuários idosos. Os resultados apresentados pela execução do teste demonstrou que botões de uso frequente apresentam distanciamento menor do considerado adequado neste estudo. Isso pode gerar baixa usabilidade devido à possibilidade de pressionar mais de uma tecla por vez, podendo gerar erros ao realizar uma tarefa.

Palavras-chave: Testes virtuais. Usabilidade. Envelhecimento. SolidWorks ${ }^{\circledR}$.

\begin{abstract}
Population-ageing comes with the necessity of assisting the elderly population abilities limitations. To achieve this it is necessary artifacts and services projects that focus on these users and their physical and cognitive condition. This paper present a proposal for the inclusion of the elderly user through virtual usability test on SolidWorks ${ }^{\circledR}$ software platform. The virtual test evaluated if the keys spacing of a remote control is adequate for elderly users. Test results showed that keys with frequent use had smaller space then what was considered adequate in this study. Lack of adequate spacing could result in low usability given the possibility of pressing more than one key at a time and might cause task error. Keywords: virtual test; usability; aging; SolidWorks ${ }^{\circledR}$.
\end{abstract}

Recebido em: 12/08/2016

Aceito em: 17/05/2018 


\section{INTRODUÇÃO}

A projeção envelhecimento populacional mundial e nacional (ANDREWS, 2000; INSTITUTO BRASILEIRO DE GEOGRAFIA E ESTATÍSTICA, 2012) traz desafios à população mundial, como o despreparo para atender as necessidades dessa população. Nichols, Rogers e Fisk (2006) destacam que a idade é relevante às considerações de design na pesquisa e prática dos fatores humanos; dentre os fatores que devem ser considerados está as diferenças críticas relacionadas à idade entre adultos jovens e idosos, que apresentam necessidades específicas de projeto.

O envelhecimento da população tende a ter uma grande influência no gerenciamento de inovação e no desenvolvimento de novos produtos em diversos segmentos da indústria. Os produtos e serviços precisam ser adaptados para melhor atender a mudança das necessidades e demandas de uma população em processo de envelhecimento (KOHLBACHER, HERSTATT, SCHWEISFURTH, 2011). As capacidades e limitações da população idosa devem ser compreendidas e abordadas no processo de design e na pesquisa de fatores humanos, buscando que esses usuários possam interagir com produtos e sistemas de maneira segura, eficiente e eficaz (NICHOLS, ROGERS, FISK, 2006).

O envelhecimento acarreta na diminuição das habilidades psicomotoras, perceptivas e cognitivas. No entanto, são poucas as iniciativas para o desenvolvimento de tecnologias que auxiliem na compensação desse declínio das habilidades (HIGGINS; GLASGOW, 2012). Nesse sentido, Haigh (1993) afirma que para ter produtos funcionais, eficientes, seguros e atraentes que aumentem as habilidades ou compensem as limitações desses usuários, faz-se necessário considerar as mudanças decorrentes do envelhecimento nos sentidos e capacidades físicas.

A usabilidade é uma área que pode auxiliar no atendimento da necessidade de entregar produtos e serviços aos usuários idosos. Segundo Nemeth (2004) um produto útil tem boa interação entre necessidade e solução, usuário e artefato. Krug (2008), de forma sucinta, coloca que a usabilidade é garantir que algo funciona bem, e que uma pessoa com habilidade e experiência comuns possa usar algo. A definição de usabilidade da NBR/ISO 9241-11 (ASSOCIAÇÃO BRASILEIRA DE NORMAS TÉCNICAS, 2011) é: "medida na qual um produto pode ser usado por usuários específicos, para alcançar objetivos específicos com eficácia, eficiência e satisfação em um contexto específico de uso". 
Uma vez que os idosos tendem não ser o foco de projeto de objetos, a usabilidade desses artefatos ao ser operados por esses usuários pode ser comprometida. A inclusão desses usuários em testes ergonômicos de usabilidade pode gerar uma melhor integração entre os idosos e os produtos presentes no mercado, sendo que terão maior compatibilidade com as limitações físicas causadas pelo envelhecimento. Uma forma para realizar a inclusão do público idoso é o uso de testes virtuais por meio de simulações. Os testes virtuais permitem a antecipação de algumas etapas de projeto de forma rápida e sem grandes custos, se comparados a testes realizados pessoalmente com os usuários.

Para atingir desempenho global, ferramentas e tecnologias de ergonomia têm evoluído para auxiliar na antecipação da análise do projeto. Uma forma para esta interação é modelagem e simulação de computador, como ocorre em áreas como a engenharia (LAUGHERY; LEBIERE; ARCHER, 2006). A modelagem digital de humanos (Digital Human Modeling - DHM) e simulação virtual de humanos foram criados para auxiliar na análise ergonômica (MA et al., 2009). Apesar de estudos terem contribuído para o desenvolvimento destas áreas, ainda não existem métodos apropriados para a modelagem humana em sistemas CAD (computer aided design) em termos de precisão de dimensões e confiabilidade de resultados (BAEK; LEE, 2012). O uso da modelagem humana em sistemas CAD envolve a construção de modelos tridimensionais humanos usando dados antropométricos publicados ou de scaners corporais, e depois avaliar interativamente se esses modelos podem realizar a atividade necessária com o artefato sendo projetado. No entanto, bases de dados convencionais não fornecem a quantidade de informações necessárias para pessoas com necessidades especiais ou aqueles que não se encaixam nos padrões, tais quais canhotos, idosos e outros (PORTER et al., 2004).

Nesse trabalho foi proposto um modelo para teste virtual de usabilidade por meio da análise do distanciamento de botões de um controle remoto utilizando a plataforma do software SolidWorks ${ }^{\circledR}$. A escolha do software se dá pelo uso disseminado entre os profissionais de design de produtos, e por apresentar ferramentas que possibilitam simular a interação entre o objeto de estudo e os usuários. Além das vantagens de custo e tempo fornecidas pelos testes virtuais, e a inclusão de um público que apresenta necessidades diferentes de um público padrão, o uso de uma plataforma conhecida dos profissionais da área de projeto de produtos pode melhorar a aplicação 
desses testes, pois os usuários do programa não necessitam de um treinamento para interagir com uma nova plataforma.

\section{DESENVOLVIMENTO - MÉTODO}

O teste virtual apresentado neste trabalho buscou verificar se o espaçamento entre os botões de um controle remoto estão adequados à realização da tarefa de manuseio pelos usuários idosos levando em consideração o dimensionamento das mãos desses usuários e suas limitações de movimento nas articulações das mãos. O distanciamento inadequado das teclas do controle pode levar a erros ao executar tarefas com o objeto, como apertar mais de uma tecla por vez, consequentemente gerar frustração do usuário pela falta de usabilidade do produto.

Strehlau, Bacha e Lora (2006) em sua pesquisa identificaram quais as atividades de lazer os idosos efetuam com maior frequência, os resultados apontaram assistir televisão como uma atividade comumente efetuada por todos participantes. Portanto a escolha do objeto de estudo foi um controle remoto.

Para a realização do teste virtual foi escolhido um modelo de controle remoto de alta veiculação e efetuado a modelagem digital do mesmo na plataforma do software SolidWorks ${ }^{\circledR}$ (Figura 1). Para a configuração do teste foi necessário coletar medidas das mãos de usuários idosos. Participaram do estudo 25 idosos (12 homens e 13 mulheres), com idade entre 60 e 85 anos. Os indivíduos aceitaram participar da pesquisa voluntariamente ao assinar ao Termo de Consentimento Livre Esclarecido (TCLE) aprovado pelo Comitê de Ética da UDESC (Número CAAE 21379913.2.0000.0118).

Figura 1 - Representação do modelo de controle remoto usado para o teste.

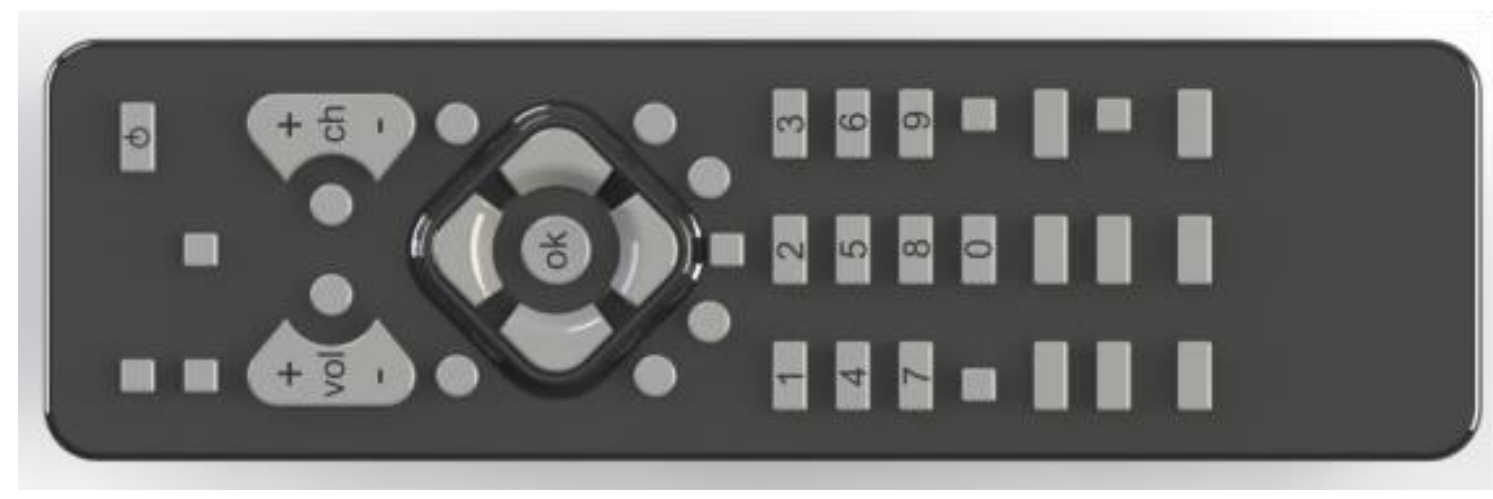

Fonte: Elaborado pelos autores. 
A configuração do teste virtual de usabilidade pela análise do distanciamento dos botões fez uso da ferramenta de tabela de projetos na modelagem do controle associada a uma macro configurada no software Excel. Macro é uma ação ou um conjunto de ações que você pode usar para automatizar tarefas (MICROSOFT, 2016). A macro tem como objetivo calcular se o distanciamento entre os botões do controle remoto está adequado para os usuários idosos. Essa distância corresponde a área que o dedo do usuário ocupa ao pressionar uma tecla, se dentro dessa área houver mais que um botão há o aumento da probabilidade de erros ao realizar uma tarefa.

Para determinar o tamanho da área livre necessária foram usadas as medidas da dimensão da ponta do dedo polegar, espessura da mão, diâmetro das articulações interfalangianas e o comprimento dos segmentos que compõe o polegar referente à mão dominante dos participantes. A Figura 2 mostra a anatomia das mãos. Essas medidas foram usadas para modelar digitalmente o polegar e simular no SolidWorks ${ }^{\circledR}$ a área de interferência dos usuários participantes em seis posições (Figura 3): com o dedo em uma posição perpendicular em relação ao controle com os ângulos de $80^{\circ}, 45^{\circ}, 35^{\circ}$ da falange distal com a superfície do controle (ângulo de ação) e três posições com o dedo angulado em relação à superfície do controle, as angulações simuladas foram de $25^{\circ}, 45^{\circ}$ e $60^{\circ}$. Os resultados foram agrupados em percentis 5,50 e $95 \%$. Por o percentil de $95 \%$ apresentar a maior área e foi adotado como a área que deve ser livre de interferência com outros botões, uma vez que usuários que apresentem área de intercessão menor terão menor dificuldade.

Figura 2 - Anatomia óssea da mão.

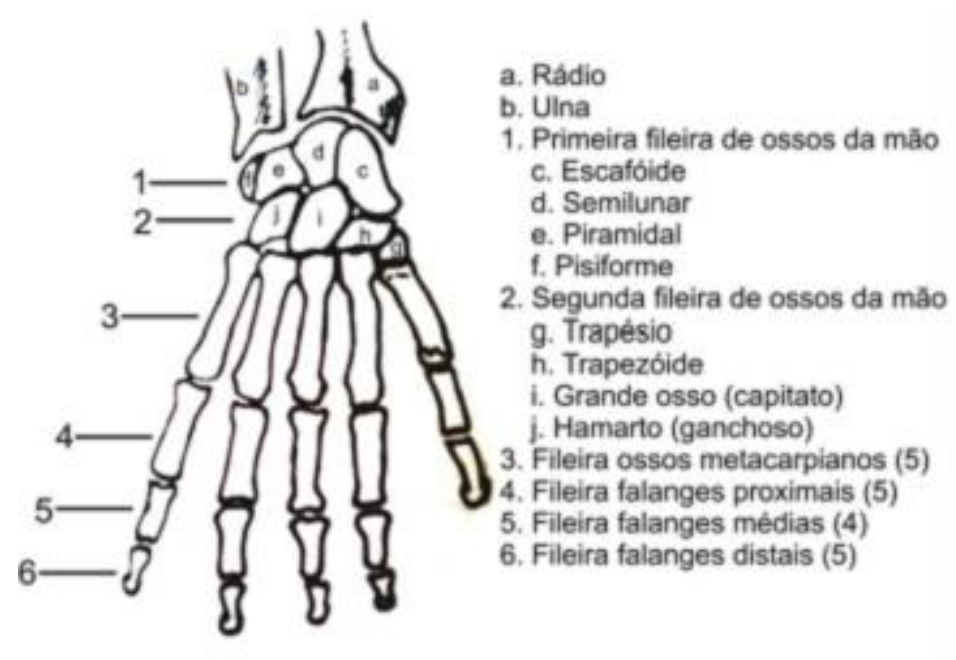

Fonte: Adaptado de Settineri (1988, p. 173). 
Figura 3 - Posições para simulação da área de interferência.

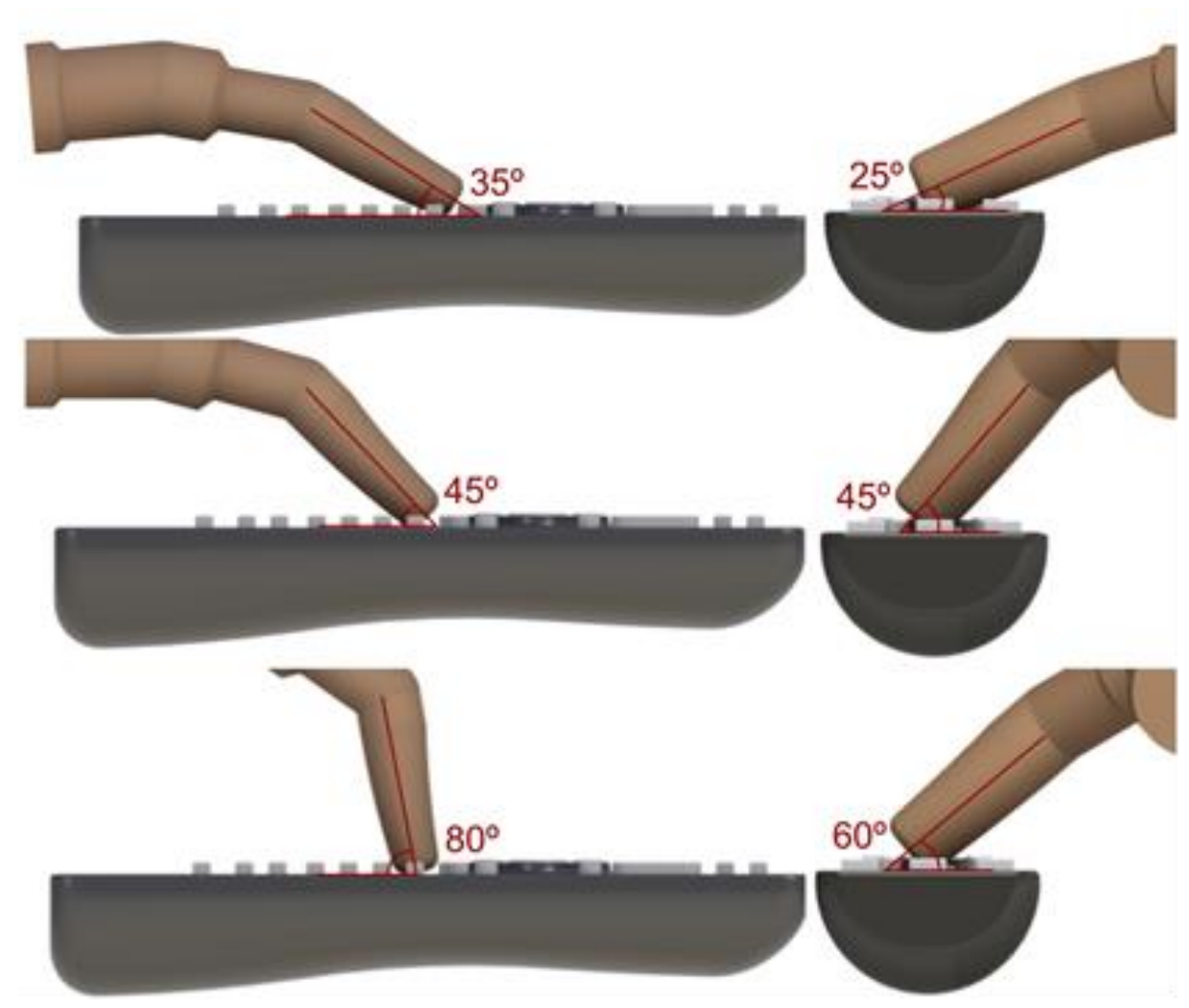

Fonte: Elaborado pelos autores.

\subsection{CONSTRUÇÃO E CONFIGURAÇÃO DO TESTE VIRTUAL}

$\mathrm{Na}$ construção do modelo virtual o dimensionamento da posição dos botões foi feito sempre do mesmo referencial para as distâncias em x (eixo horizontal) e em y (eixo vertical), o ponto para referência dessas dimensões no botão foi o ponto central. Também foi desenhado um círculo, com área correspondente à área de contato determinada, concêntrico a cada botão por linha de projeção, esse círculo foi projetado na superfície do controle, e as linhas de projeções foram suprimidas. Para não gerar complicações nos cálculos os botões que não apresentavam formato retangular ou circular foram simplificados para adotar essas características, mas a posição do centro desses botões foi mantida. A simplificação da forma desses botões tem pouco impacto na interpretação dos dados por as áreas suprimidas para a simplificação da forma são áreas inoperantes do controle.

$\mathrm{Na}$ aba de gerenciamentos de configurações do arquivo é adicionada uma configuração filha da configuração principal, com o nome resultados. Na configuração resultado serão escritos os 
resultados da operação da macro. Após gerar a tabela de projetos com todas as informações da construção do modelo e o estado de exibição das features, essa tabela é salva num arquivo Excel, fora do SolidWorks ${ }^{\circledast}$ (SW). Nessa tabela são organizados os dados de forma que, as informações dos botões estejam agrupadas em sequência. As informações de cada botão ocupam seis colunas: distância do centro em x, distância do centro em y, largura do botão ou diâmetro, altura do botão ou para botões circulares uma coluna em branco, o estado de exibição da área livre de interferências desse botão, e por fim, o diâmetro do círculo de área livre do botão.

No Excel foi salvo um arquivo com a função de macro em que ao executar a macro esta abre o arquivo da tabela de projeto; calcula as distâncias entre os botões; nos botões em que a distância não está adequada, modifica na linha de resultados a condição do estado de exibição do círculo da área livre mínima para aparente; salva o arquivo da tabela de projetos e fecha o arquivo. Para definir se os botões estão devidamente espaçados, a macro calcula a distância entre centros de dois botões. Essa distância é então comparada à soma do raio da área definida como área mínima por botão, com o raio ou metade da largura de um botão retangular dos dois botões sendo analisados (Equação 1). Caso a soma de um ou dos dois botões analisados for menor que a distância encontrada, o distanciamento entre botões não está adequado. A programação da macro pode ser visualiza na Figura 4.

Equação 1 - Condição para determinar distanciamento adequado.

$$
\begin{gathered}
r_{\text {area livre min. }}+r_{\text {botä̀ }} \\
\text { ou } \\
r_{\text {ấrea livre min. }}+L / 2
\end{gathered}
$$

Fonte: Elaborado pelos autores.

Figura 4 - Programação da macro.

Workbooks.

Open Filename:="C:Itabela_c_T2.xIsx"

Range("F3:KN3").Select

Selection.Copy 


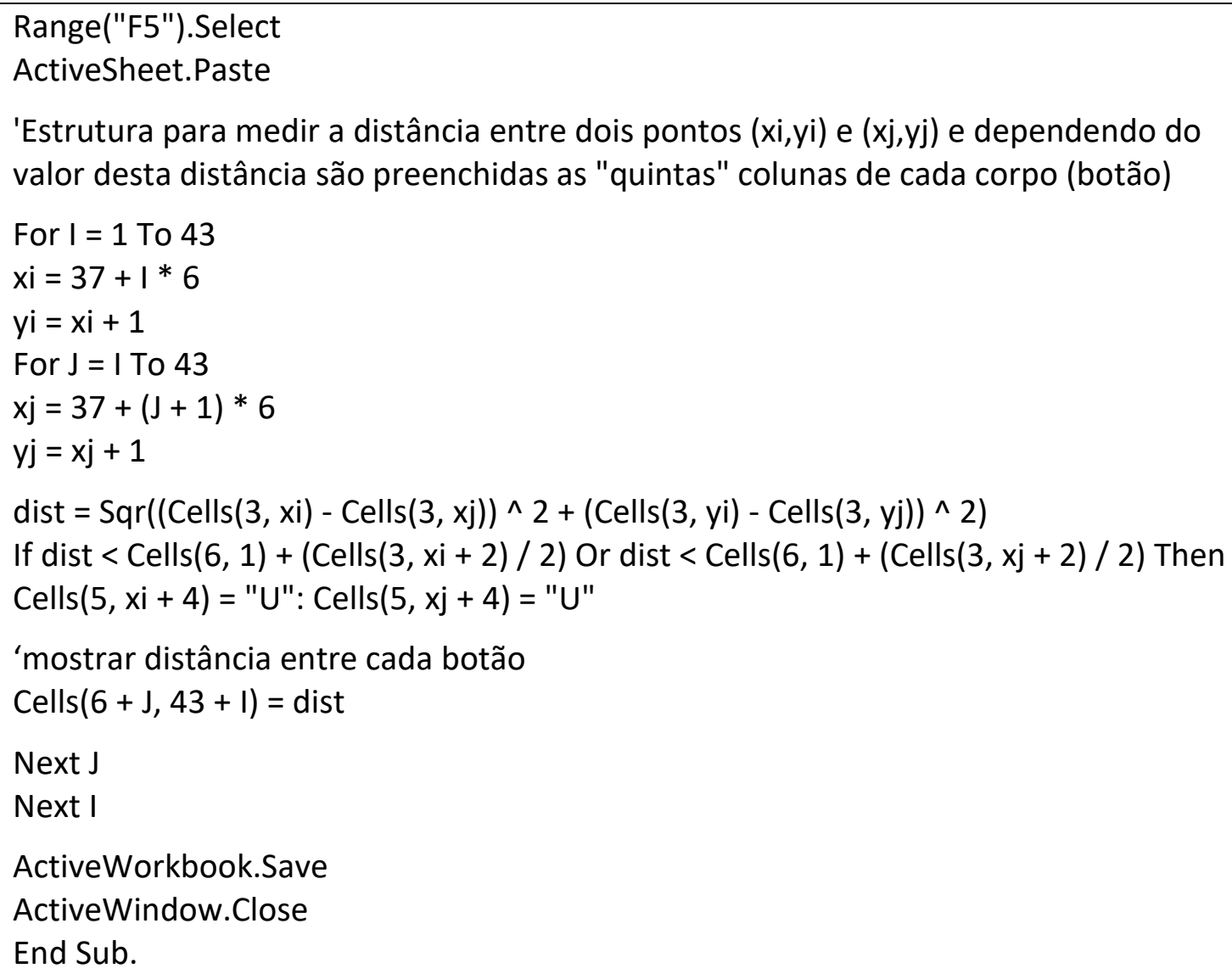

Fonte: Elaborado pelos autores.

Para que os resultados sejam visualizados no SolidWorks ${ }^{\circledR}$ é necessário substituir a tabela atual do arquivo pela tabela de projeto do arquivo salvo pela macro e habilitar a configuração resultados. Essa atividade permite a visualização na modelagem do controle os botões que apresentam algum tipo de conflito, esses botões terão um círculo correspondente à área livre mínima determinada.

\section{RESULTADOS}

A partir das medidas antropométricas coletadas com os usuários idosos foi calculada a área de interferência entre o dedo destes usuários e o controle remoto (Tabela 1). Essas medidas foram aferidas de forma indireta no software SolidWorks ${ }^{\circledR}$ com uso de ferramentas de interseção do software. 
Tabela 1 - Área de contato em posições determinadas - $\mathrm{mm}^{2}$ (referente à Figura 3).

\begin{tabular}{lccccc}
\hline \multirow{2}{*}{ Medidas } & \multicolumn{3}{c}{ Percentis } & \multirow{2}{*}{ Média } & \multirow{2}{*}{ DP } \\
\cline { 2 - 4 } & $\mathbf{5 \%}$ & $\mathbf{5 0 \%}$ & $\mathbf{9 5 \%}$ & & \\
\hline área 80 (dedo perp.) & 46,12 & 66,25 & 104,58 & 67,65 & 13,76 \\
área 450 (dedo perp.) & 29,55 & 37,09 & 67,75 & 38,78 & 9,00 \\
área 35 (dedo perp.) & 30,94 & 37,68 & 47,31 & 38,42 & 4,59 \\
área 25 (dedo rot.) & 21,17 & 24,44 & 31,96 & 25,39 & 3,17 \\
área 45 (dedo rot.) & 16,21 & 19,55 & 24,48 & 19,91 & 2,69 \\
área 60 (dedo rot.) & 16,04 & 20,53 & 26,02 & 20,42 & 3,17 \\
\hline
\end{tabular}

Fonte: Elaborado pelos autores.

Para realizar a análise do distanciamento de botões por meio do teste virtual proposto, foi definida como área mínima livre entre os botões uma área de tamanho $104,56 \mathrm{~mm}^{2}$, representado em uma área circular de diâmetro de $11,52 \mathrm{~mm}$, maior área encontrada no percentil $95 \%$ dos usuários.

Ao executar a macro e carregar a tabela do projeto resultante, e habilitando a configuração "resultados" no software, o resultado atingido é o apresentado na Figura 5.

Figura 5 - Resultado da análise virtual de distanciamento de botões.

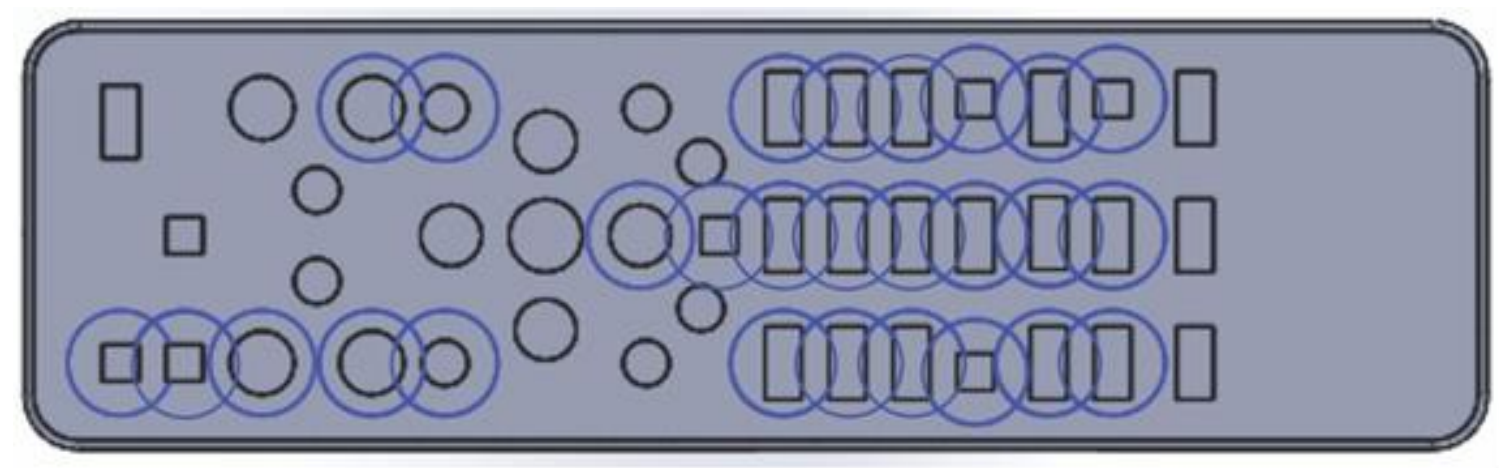

Fonte: Elaborado pelos autores.

Analisando a Figura 4 pode-se perceber que o distanciamento entre os botões numéricos e demais botões da parte inferior do controle remoto não apresentam um distanciamento adequado para os usuários idosos. Alguns botões de maior uso como volume (aumentar ou diminuir) e canal para baixo também foram identificados com pouco espaçamento entre botões. Esses botões apontados na análise podem levar a um erro na execução de tarefas com o controle remoto, pois 
pode ocorrer que o usuário pressione mais de um botão ao efetuar uma atividade com o objeto.

\section{CONCLUSÃO}

Avaliações virtuais como a apresentada nesse artigo, com o uso da ferramenta de tabela de projetos e com a realização de uma análise pela execução de uma macro no software Excel se mostrou ser uma alternativa viável para as análises iniciais de um projeto. Essa avaliação apresenta custos mínimos e pode apresentar resultados para ajustar possíveis problemas no uso do produto antecipadamente no processo de desenvolvimento do produto. Pelo resultado o projetista pode readequar a distribuição de botões para melhor interação do produto com os usuários, antes mesmo da confecção de um modelo físico do produto.

A avaliação virtual de distanciamento entre botões, realizada pela execução de uma macro, demonstrou que grande parte do controle remoto avaliado apresenta um espaçamento entre botões menor do que foi estimado como o espaçamento mínimo para esses usuários. O espaçamento inadequado dos botões leva a suposição de que o uso deste controle remoto apresentaria um alto índice de insucesso da realização da tarefa ou desconforto. No entanto esse resultado em um cenário real pode apresentar resultados diferentes uma vez que o comportamento de uso do objeto é variável de usuário para usuário. Dependendo da forma que o dedo de acionamento chega ao botão ele irá imprimir uma área de contato diferente, pelo comportamento de uso, pode ser que cada conjunto de teclas do controle apresente uma área mínima de distanciamento entre botões diferentes, assim, afetando o resultado da análise, também devem ser consideradas a altura das teclas, a pressão e o deslocamento necessários para seus acionamentos.

\section{AGRADECIMENTOS}

O presente trabalho foi realizado com apoio do CNPq, Conselho Nacional de Desenvolvimento Científico e Tecnológico e CAPES, Coordenação de Aperfeiçoamento de Pessoal de Nível Superior. 


\section{REFERÊNCIAS}

ANDREWS, Gary. Los desafíos del proceso de envejecimiento en las sociedades de hoy y del futuro. In: ENCUENTRO LATINOAMERICANO Y CARIBEÑO SOBRE LAS PERSONAS DE EDAD, 1999, Santiago. Anais... Santiago: CELADE, p. 247-256, 2000.

ASSOCIAÇÃO BRASILEIRA DE NORMAS TÉCNICAS. NBR 9241-11: Requisitos ergonômicos para o trabalho com dispositivos de interação visual. Parte 11 - Orientações sobre usabilidade. Rio de Janeiro, 2011.

BAEK, Seung-yeob; LEE, Kunwoo. Parametric human body shape modeling framework for humancentered product design. Computer-Aided Design, S.I., v. 44, n. 1, p.56-67, jan. 2012. Disponível em: <https://www.sciencedirect.com/science/article/pii/S0010448510002289>. Acesso em: 08 out. 2018.

HAIGH, Ruth. The ageing process: a challenge for design. Applied Ergonomic, S.I., v. 24, n. 1, p.9-14, fev. 1993. Disponível em:

<https://www.sciencedirect.com/science/article/pii/000368709390153Z?via\%3Dihub>. Acesso em: 08 out. 2018.

HIGGINS, Peter G; GLASGOW, Adam. Development of guidelines for designing appliances for older persons. Work, S.I., v. 41, n. 1, p.333-339, jan. 2012. Disponível em:

<https://content.iospress.com/download/work/wor0178?id=work\%2Fwor0178>. Acesso em: 08 out. 2018.

INSTITUTO BRASILEIRO DE GEOGRAFIA E ESTATÍSTICA. Séries estatísticas \& Séries históricas. Série: POP22 - População por grupos de idade (população presente e residente). 2012. Disponível em: $<$ http://seriesestatisticas.ibge.gov.br/series.aspx?no=10\&op=0\&vcodigo=POP22\&t=populacaogrupos-idade-populacao-presente-residente>. Acesso em: 08 out. 2018.

KOHLBACHER, Florian; HERSTATT, Cornelius; SCHWEISFURTH, Tim. Product development for the silver market. In: KOHLBACHER, Florian; HERSTATT, Cornelius. The Silver Market Phenomenon: marketing and innovation in the aging society. Berlin: Springer, 2011. p. 3-13.

KRUG, Steve. Não me faça pensar: uma abordagem de bom senso à usabilidade na web. 2. ed. Rio de Janeiro: Alta Books, 2008.

LAUGHERY, K. Ronald; LEBIERE, Christian, ARCHER, Susan. Modeling Human Performance in Complex Systems. In: SALVENDY, Gavriel. Handbook of Human Factors and Ergonomics. Hoboken, NJ: John Wiley \& Sons, 2006. Cap. 36, p. 965-996.

MA, Liang et al. A new simple dynamic muscle fatigue model and its validation. International Journal Of Industrial Ergonomics, [s.I.], v. 39, n. 1, p.211-220, jan. 2009. Disponível em: <https://www.sciencedirect.com/science/article/pii/S0169814108000863>. Acesso em: 08 out. 2018. 
MICROSOFT. Excel 2016 Ajuda: Executar uma macro, 2016.

NEMETH, Christopher. P. Human Factors Methods for Design: Making Systems Human-Centered. Boca Raton, FL: CRC Press, 2004.

NICHOLS, Timothy A.; ROGERS, Wendy A.; FISK, Arthur D. Design for Aging. In: SALVENDY, Gavriel. Handbook of Human Factors and Ergonomics. Hoboken, NJ: John Wiley \& Sons, 2006. Cap. 54, p. 1418-1445, 2006.

PORTER, J.mark et al. 'Beyond Jack and Jill': designing for individuals using HADRIAN. International Journal of Industrial Ergonomics, [s.I.], v. 33, n. 3, p.249-264, mar. 2004. Disponível em: <https://www.sciencedirect.com/science/article/pii/S016981410300146X>. Acesso em: 08 out. 2018.

STREHLAU, Vivian Iara; BACHA, Maria de Lourdes; LORA, Maíra Ivanoff. Idosos não são iguais: uma análise de agrupamentos sobre as atividades de lazer da terceira idade. In: EMA - ENCONTRO DE MARKETING, 2., 2006, Rio de Janeiro. Anais... . Rio de Janeiro: Anpad, 2006. p. 1 - 15. Disponível em: <http://www.anpad.org.br/admin/pdf/ema2006-mktc-230.pdf>. Acesso em: 08 out. 2018.

SETTINERI, Luiz Irineu Cibils. Biomecânica: noções gerais. Rio de Janeiro: Atheneu, 1988. 